\title{
Laboratory tests on the cleanliness of soil materials used as subgrades in pavement structures
}

\author{
A. Athanasopoulou \& G. Kollaros \\ Democritus University of Thrace, Greece
}

\begin{abstract}
In order to ascertain the presence of very fine material passing the No. 200 sieve, cleanliness tests are performed in the laboratory. The most important of these tests refer to the determination of the quantity of fines, of the sand equivalent value, of the plasticity index, as well as the methylene blue value of the material tested. Performing the sand equivalent test the percentage of very fine dust in claylike form can be established. Materials with very low sand equivalent are characterized as "impure" and the possibility of the existence of clay size grains in them is very high. In such cases it is necessary to perform a test in order to determine the plasticity of the soil material. The determination of the plasticity index of natural soils specifies their suitability as subgrade layers in pavement structures. Soils having a sand equivalent value lower than $10 \%$ will develop excessive swell with a simultaneous decrease in their bearing capacity when a pavement is built on them. The quantity of methylene blue absorbed by clay components of a soil mass is proportional to the specific area of the clay minerals. The methylene blue test supplements the sand equivalent and Atterberg limits tests, since with these the existence of clay-size grains is determined, but not the existence of active clay minerals as well. A number of soil samples have been examined in the laboratory, involving the procedures of cleanliness tests. The quantity of fine grained material was determined and their activity was accessed. The magnitudes were correlated in order to check the suitability of soils as a pavement foundation. Empirical relationships have been established which connect the attributes characterizing the cleanliness of soil materials. These relationships compare very well with most of the findings published worldwide.
\end{abstract}

Keywords: swelling soils, methylene blue test, sand equivalent, cleanliness. 


\section{Introduction}

In most pavement structure techniques, suitability is one of the essential characteristics of soil materials. The term "suitability" characterizes the absence of undesirable elements. These elements are soluble salts, iron hydroxides, organic matter, as well as clays. They can present sickliness because of their fineness, their physicochemical activity or because of their effect in the binding materials. In view of their frequency of appearance in alluvial deposits and rocks, their hydrophilic nature, and of their affinity and plasticity properties, clays are the most harmful.

Swelling soils often cause serious damages in pavement structures, while, on the other hand, they cannot be used as embankment material in roads. The swelling potential of soils is mainly affected by their clayey fraction (Kollaros and Athanasopoulou [1]). In order to ascertain the existence of very thin material passing the No. 200 sieve cleanliness tests are performed. The more important tests are consisted in the determination of the quantity of fines, of the plasticity index, the equivalent sand and the methylene blue value.

The quantity of material passing the $0,075 \mathrm{~mm}$ sieve (\#No. 200), expressed as a percentage of the total material, is determined by the sieve analysis test, either through the dry or, more usually, through the wet process, according to AASHTO T88 or ASTM D422 standards.

The plasticity index, which is defined as the difference in contained moisture between the liquid and plasticity limits: PI=LL-PL, it is a value that helps to recognize soil characteristics and to properly classify them. The plasticity index determines the appropriateness of soils to be used as subgrades in roadway foundations. Soils having a high PI value, such as clayey, silty and some sandsilt soils, are inadequate for the foundation of pavements. In Table 1 the degree of plasticity is given in terms of PI and of soil characteristics in a dry condition, for the corresponding scale of the PI values.

The plasticity index is useful for materials containing high percentages of silt and clay, while in the region not covered by the PI use is made of the sand

Table 1: $\quad$ Soil characterization as a function of PI.

\begin{tabular}{|c|c|c|}
\hline PI & Soil & Soil characteristics in dry condition \\
\hline$>35$ & $\begin{array}{l}\text { Highly } \\
\text { Plastic }\end{array}$ & $\begin{array}{l}\text { High cohesion value, manual smashing of lumps is } \\
\text { impossible }\end{array}$ \\
\hline $16-35$ & Plastic & $\begin{array}{l}\text { Medium to high cohesion, difficulty in smashing lumps } \\
\text { manually }\end{array}$ \\
\hline $7-15$ & $\begin{array}{l}\text { Medium } \\
\text { plasticity }\end{array}$ & $\begin{array}{l}\text { Low to medium cohesion, lumps are smashed by exerting } \\
\text { a low pressure to them }\end{array}$ \\
\hline $4-6$ & $\begin{array}{l}\text { Slightly } \\
\text { plastic }\end{array}$ & Low cohesion, easy manual smashing of lumps \\
\hline $0-3$ & Non Plastic & $\begin{array}{l}\text { Very little cohesion or complete absence of cohesion, } \\
\text { lumps are decomposed by a simple touch }\end{array}$ \\
\hline
\end{tabular}


equivalent value. The sand equivalent, $\mathrm{SE}$, test arose in view of the need for a quick quality indication of paving materials. This test was devised by Hveem in 1953, it was put in use in 1956 and it has been adopted for laboratorial use since 1966.

More specifically, with the sand equivalent test the proportion of very fine dust of claylike form is determined. For a $\mathrm{SE}<20$ value, the plasticity index should be determined. In the region of higher SE values, the plasticity index is not determined (Non Plastic) and therefore it cannot replace the sand equivalent.

On the basis of comparisons of sand equivalent values to the results of other tests it has been found that most of the soils presenting high swell potential when saturated with water, is possible to be recognized by the SE value. Soils on top of which a pavement is to be constructed develop excess swell, with a simultaneous decrease of their bearing capacity in saturated conditions, provided that the SE value is lower than $10 \%$. The different fine grained materials could be classified in three groups, as shown in Table 2, depending on their sand equivalent value.

Table 2: $\quad$ Classification of fine-grained materials as a function of SE.

\begin{tabular}{|c|c|}
\hline Sand Equivalent & Fine-grained materials \\
\hline High Values & Clean non-cohesive sands \\
\hline Medium Values & Sands blended with some quantities of silt and clay \\
\hline Low Values & Clays and silt-clayey mixtures \\
\hline
\end{tabular}

In order to measure the unsoundness of sands, the Laboratoire Centrale des Ponts et Chaussées, in France, examined two different methods. According to the first procedure, the process of the sand equivalent test was modified, to become more compatible for the recognition of sands rich in fine-grained material. Thus, in the AFNOR NF P 18-597 standard, "Propretés des Sables", the following modifications were made:

- $\quad$ Test sampling using the $2 \mathrm{~mm}$ sieve, instead of the $4.75 \mathrm{~mm}$ sieve (\#No. 4).

- The percentage of elements finer than $0.08 \mathrm{~mm}$ is kept to a constant value equal to $10 \%$.

With the second procedure, the test is supplemented with a different measure, namely the capacity of the clayey components to absorb some basic dye such as the methylene blue.

The methylene blue test has basically been developed in an effort to determine the clay content of a soil. In 1980 the test was adopted for aggregates and in 1984 the method of methylene blue with agitation was proposed by Tran and Millon-Devigne [2] in order to increase the precision of the test and to make it capable of measuring properties on least clayey samples.

The test is based on a successive import of increasing quantities of methylene blue solution in a suspension of the material to be examined, until the claylike particles are saturated with dye. Saturation has been achieved when a spot of soil coloured by the retained dye of soil is shaped, surrounded from a colourless area of humidity ("spot" control) on a filter paper. The control is positive, if in this zone an excess of dye appears in the form of light blue coloration, which surrounds 
radially the central dark blue trace of the drop. The spot test is repeated after two minutes. If the solution continues to demonstrate an excess of dye, the "final point" has been reached. It must be noted that an experienced person can reach faster the final point, omitting some initial doses of methylene solution.

The methylene blue test supplements the sand equivalent and Atterberg limits tests, since they determine the existence of particles with claylike dimensions, but not the presence of active clay minerals.

\section{Laboratory testing of soil samples}

Soil samples were collected from twenty six sites located in the Evros prefecture. The criterion used was their proximity to road works and the probability that they could create constructional problems due to large percentages of clay. The properties of these materials ranged in the limits presented in Table 3.

Table 3: $\quad$ Properties of the soils tested.

\begin{tabular}{|c|c|c|}
\hline Soil Property & Mean Value & Range of values \\
\hline Clay content $($ material $<2 \mu)(\%)$ & 41.42 & $9-54$ \\
\hline Percentage passing the No. 200 sieve $(\%)$ & 74.65 & $29.23-90.13$ \\
\hline Liquid Limit $(\%)$ & 52.73 & $29-76$ \\
\hline Plasticity Limit $(\%)$ & 22.24 & $17-29$ \\
\hline Plasticity Index $(\%)$ & 29.73 & $17-47$ \\
\hline Linear shrinkage $(\%)$ & 12.93 & $10.0-15.7$ \\
\hline Volume of dye, $\mathrm{V}_{\mathrm{d}}\left(\mathrm{cm}^{3}\right)$ & 20.35 & $6-27.5$ \\
\hline Sand equivalent, SE & 14.46 & $5-33$ \\
\hline Skempton Activity [PI/ $(\%$ material $<2 \mu)]$ & 0.71 & $0.58-1.00$ \\
\hline
\end{tabular}

The swelling potential of soils is influenced by the percentage of the clay fraction they contain as well as by the composition of this fraction. The grain size analysis of soil samples revealed that most of them had high content of clay (material with grain diameter smaller than 2 microns).

When the grain size analysis was performed using sieves with standard openings, the presence of high percentages of very fine material passing the No. 200 sieve has been recorded. Only two samples had particles with a diameter smaller than $0,075 \mathrm{~mm}$ in a percentage smaller than $50 \%$.

The Atterberg limits tests resulted in high values for the plasticity index. The PI values were above 15 for all soil samples, reaching even a value of 47, suggesting that the soils were very plastic and in a dry condition it was very difficult to smash any formed lump. The determination of PI was not possible for one of the samples. Soils having such PI values are inadequate for the foundation of pavements.

The swelling degree could be calculated with the Holtz-Gibbs classification method, which is based on the plasticity index and the colloid content of the samples. Twenty two samples are characterized by a very high or high swelling degree, while only 4 showed medium and low swelling degree. Generally, the 
clays in the region fall in group A-7 according to the AASHTO classification method. This group involves soil materials poor or unsuitable to be used as foundation.

\section{Correlation of attributes used for soil characterization}

With the completion of laboratory testing, a statistical correlation of attributes used for the characterization of soil materials was attempted. Special attention has been focused mainly on those values resulting from the test which specifies the clayey ingredients using the methylene blue and the sand equivalent procedures. The selection of these properties can be easily justified taking into account the high percentages of fine grained material in the samples and the relatively high swelling degrees that are expected in the field, constituting a source of dangers for the integrity and longevity of pavement structures.

The sand equivalent values resulted as the mean of three repetitions of the standard process for each sample. In the methylene blue test the volume of dye, $\mathrm{V}_{\mathrm{d}}$ was measured.

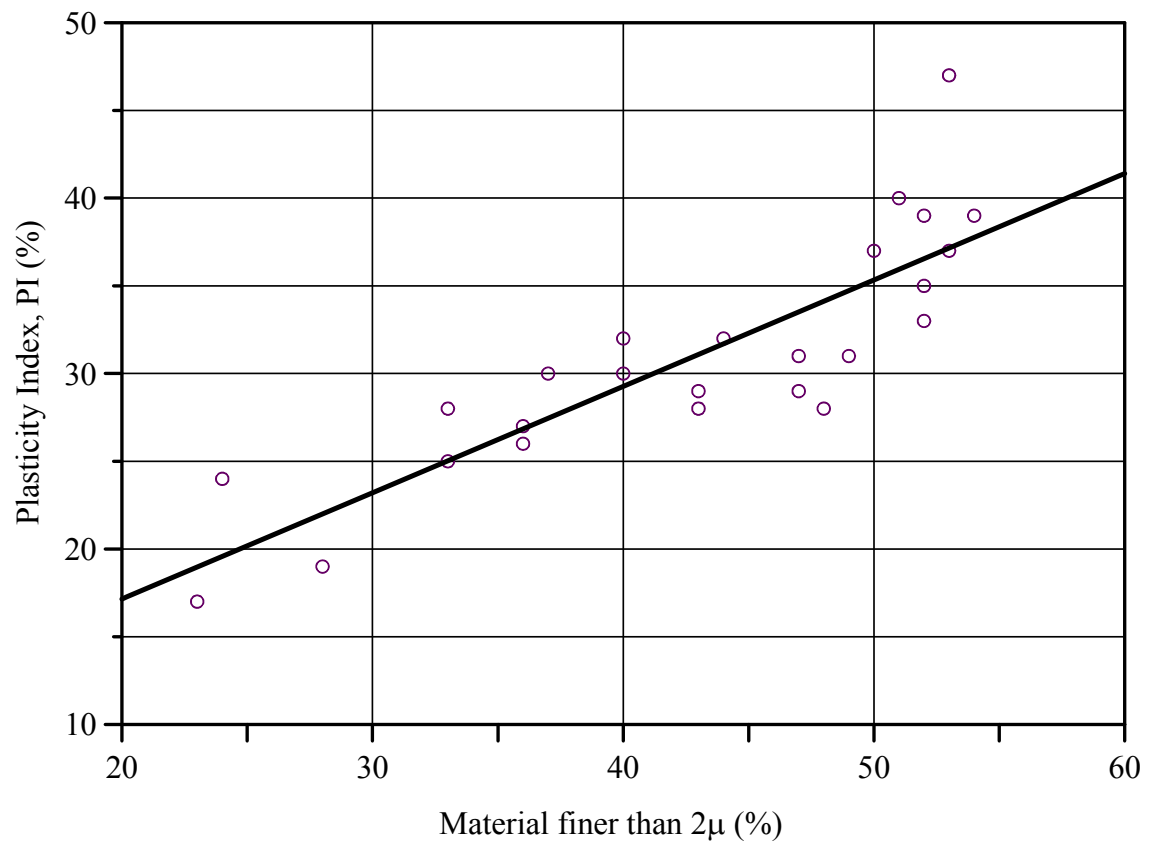

Figure 1: $\quad$ Plasticity index variation with material finer than $2 \mu$.

The distribution of plasticity index values, as estimated by the percentage of material finer than $2 \mu$ as well as from the results of the methylene blue test is given in figures 1 and 2, respectively. The linear correlation of these attributes with the plasticity index is particularly good, since the coefficients of determination in these 
cases are $\mathrm{R}^{2}=0,73$ and $\mathrm{R}^{2}=0,58$. Equations (1) and (2) interrelate the results obtained for the plasticity index and the other two properties.

$$
\begin{gathered}
\mathrm{PI}=0,606(\% \text { material }<2 \mu)+5.02 \\
\mathrm{PI}=1,201 \mathrm{~V}_{\mathrm{d}}+5.79
\end{gathered}
$$

In particular, a high degree of correlation has been obtained for the methylene blue value and the percentage of claylike fraction in the samples tested. The linear regression eqn. (3) connecting these attributes gave a coefficient of determination, $\mathrm{R}^{2}$, equal to 0,82 .

$$
\% \text { material }<2 \mu=2.019 \mathrm{~V}_{\mathrm{d}}+0.34
$$

In figure 3 the variation of the percentage of material with particle diameter smaller than $2 \mu$ against the Volume of dye calculated by the methylene blue method is presented.

A similarly good linear correlation appeared between the volume of dye of the samples and the percentage of material passing the sieve No. 200, as shown in figure 4 . The coefficient of determination, $\mathrm{R}^{2}$, for the relation connecting the two attributes in eqn. (4) has been found equal to 0,69 .

$$
\% \text { material }<\text { No. } 200=2.409 \mathrm{~V}_{\mathrm{d}}+25.63
$$

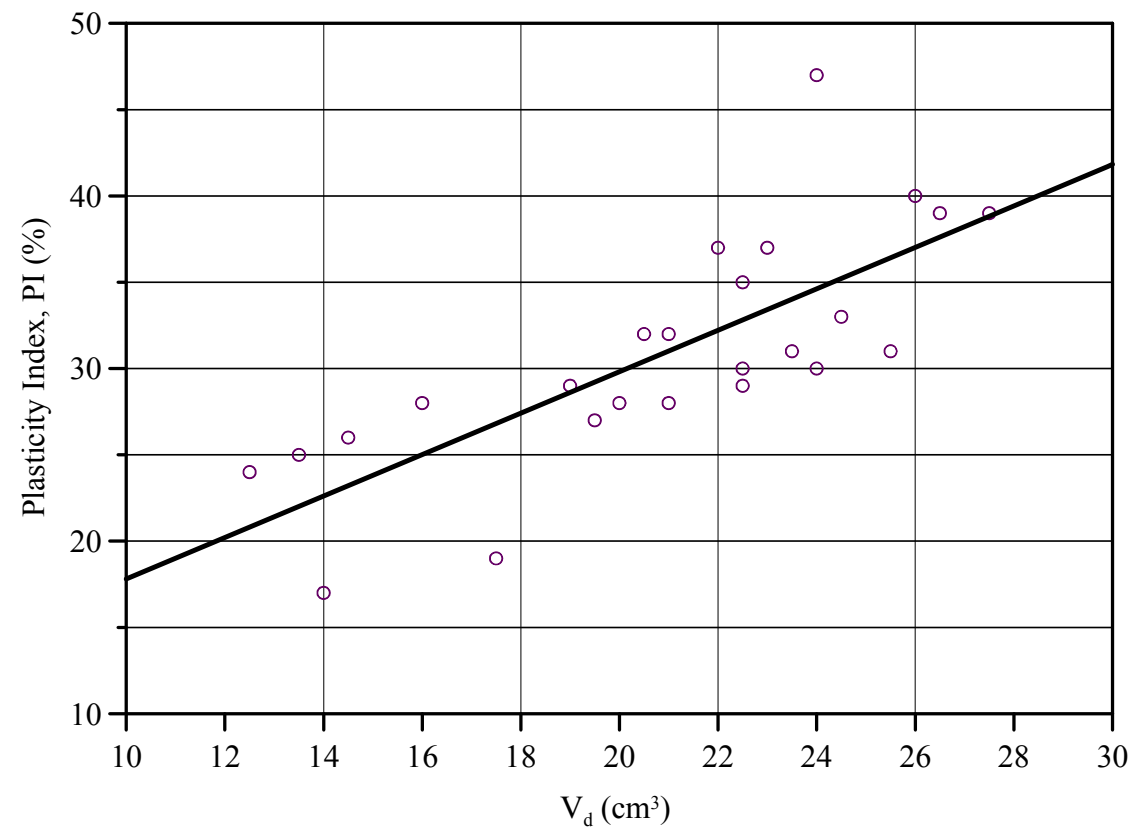

Figure 2: $\quad$ Plasticity index variation with volume of dye, $\mathrm{V}_{\mathrm{d}}$. 


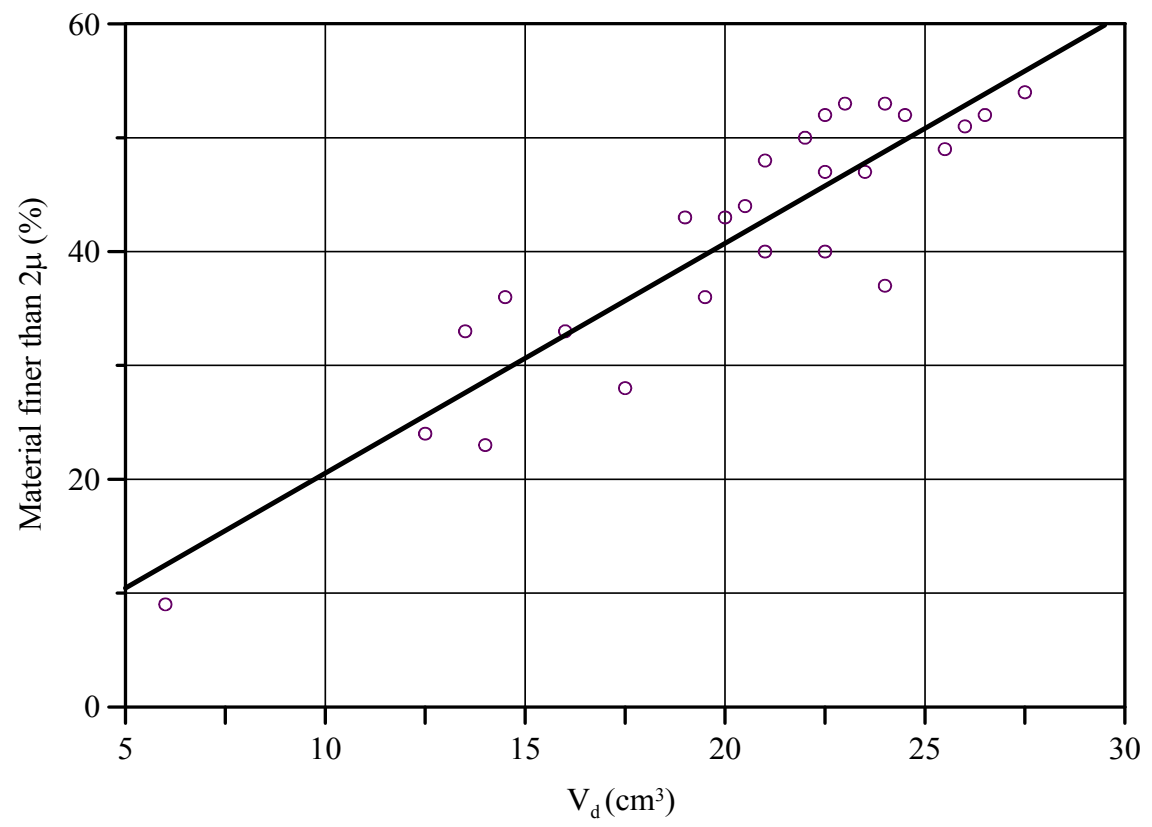

Figure 3: $\quad$ Variation of the percentage of material $<2 \mu$ as a function of the $V_{d}$.

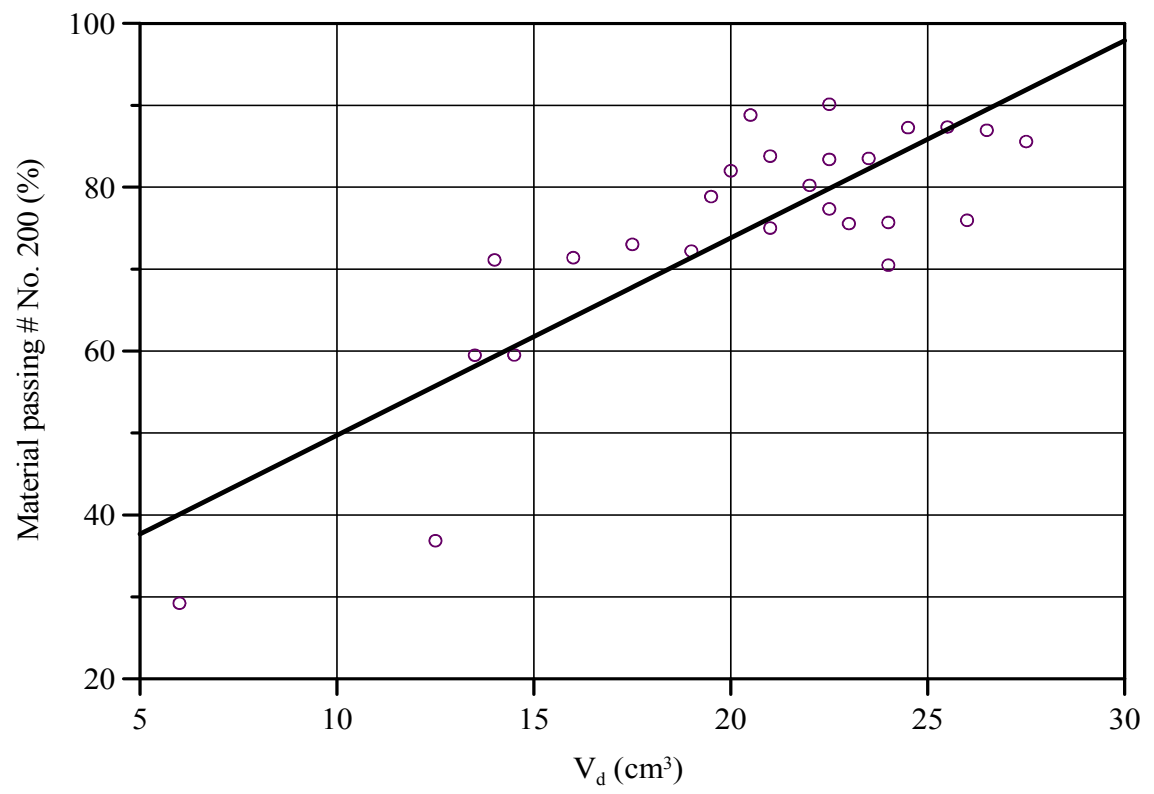

Figure 4: Variation of material passing \# No. 200 as a function of the Volume of dye, $\mathrm{V}_{\mathrm{d}}$. 


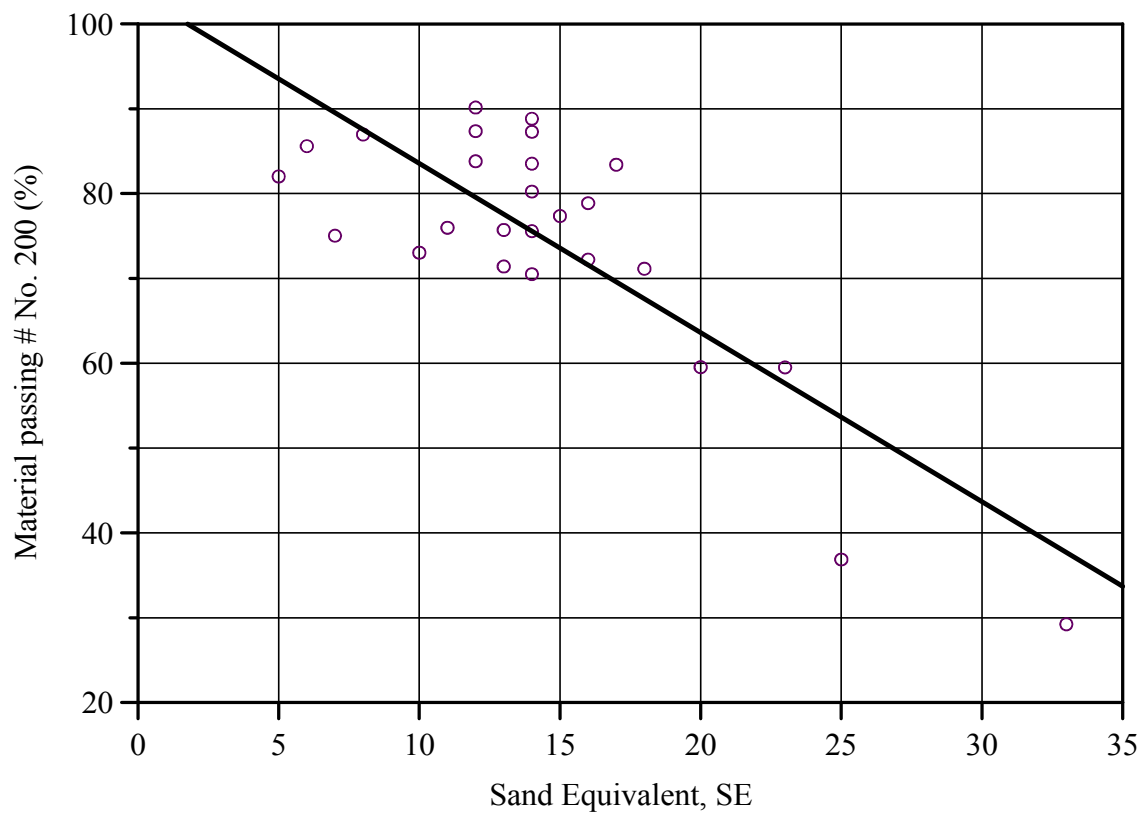

Figure 5: Variation of the percent passing \# No. 200 as a function of the sand equivalent value.

In eqn. (5) the sand equivalent value, $\mathrm{SE}$, is the independent variable and the percentage of material passing the sieve No. 200 is the dependent variable. This equation yielded a coefficient of determination, $\mathrm{R}^{2}=0,66$.

$$
\% \text { material }<\text { No. } 200=1.994 \mathrm{SE}+103.48
$$

The sand equivalent values have been related to the respective values obtained by the testing of the samples using the methylene blue test. The equation of the curve that fits to the data in figure 6 has a coefficient of determination, $\mathrm{R}^{2}=0,43$ and is depicted in eqn. (6).

$$
\mathrm{V}_{\mathrm{d}}=-0,587 \mathrm{SE}+28.98
$$

The negative gradient of the regression lines in figures 5 and 6 means that an increase of the sand equivalent entails a reduction in the respective values of both the fine-grained material and the quantity of dye absorbed by the clay particles.

Empirical relations similar with those resulted from the statistical processing of the laboratorial results, have been reported in the international bibliography. Many research efforts have been devoted to the correlation of the Atterberg limits with the attributes tested and with the cation exchange capacity, CEC. High level of correlation $(\mathrm{r}=0,80)$ has been obtained between the plasticity index and the CEC (Taylor [3]). Also, a coefficient $\mathrm{R}^{2}=0,56$ was found for the relation of the Skempton activity $(\mathrm{PI} / \%$ of material $<2 \mu)$ with the cation exchange 


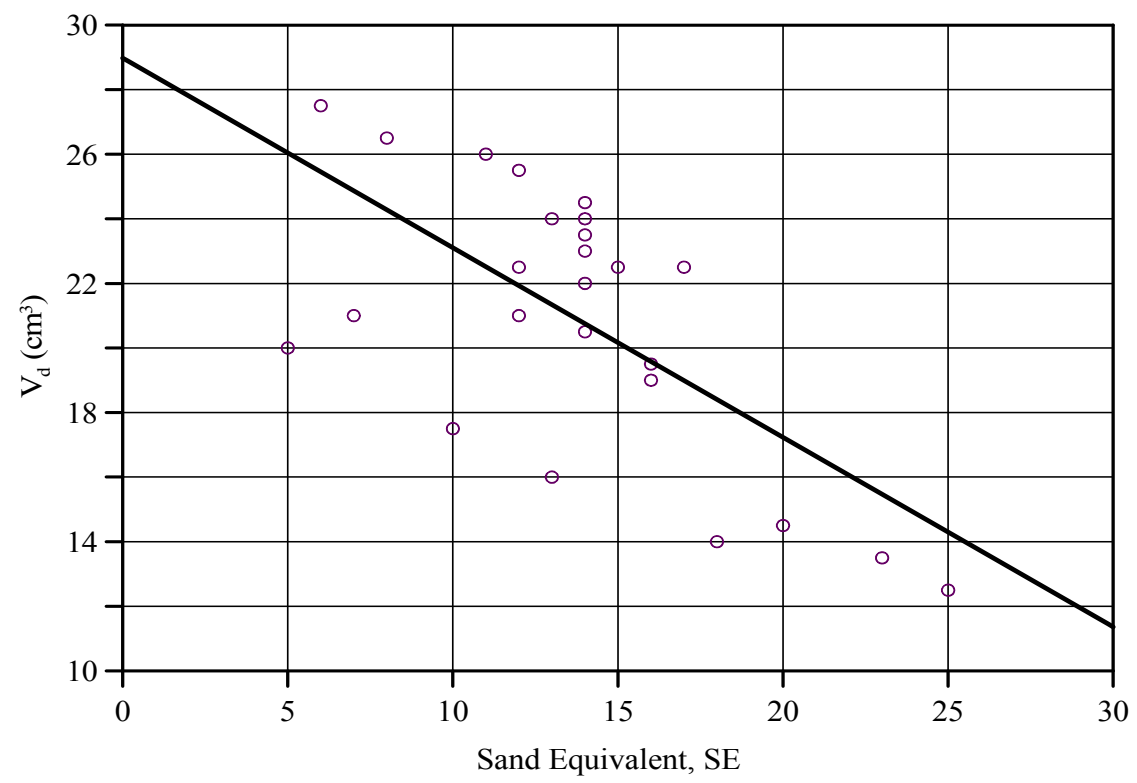

Figure 6: Variation of $\mathrm{V}_{\mathrm{d}}$ as a function of the sand equivalent value.

capacity (Sweere and Galjaard [4]). The plasticity index of 70 Illinois clay samples (Odell et al. [5]) was fairly correlated with the content of material $<2 \mu$ through the eqn. (7):

$$
\mathrm{PI}=0,568(\% \text { material }<2 \mu)+1.09
$$

A very good correlation between the apparent surface area calculated by the methylene blue test and the percentage of particles having a diameter smaller than $1 \mu$ has been found by Xeidakis [6]. In this research 8 clayey minerals had been tested and a correlation coefficient $\mathrm{r}=0,946$ was found. A relatively weaker correlation $(\mathrm{r}=0,65)$ has been reported for 19 British soils (Farrar and Coleman [7]) which contained some montmorillonite.

Nikolaides et al. [8] published the results of SE and MB values obtained at 16 samples from Greek quarries. They found that there is no correlation between $\mathrm{MB}$ and SE values.

The results of a series of tests performed on aggregates from production sites and 45 mixtures prepared in the laboratory to clarify the effect of potentially harmful fines on the MB and SE values are described by Petkovšek et al. [9]. Particular attention is paid to the prediction of approximate limits for SE and MB values, taking into account the specific geological conditions of the aggregate sources and past experience using the same aggregates. 


\section{Conclusions}

Swelling clay soils are abandoned in the region of Thrace, Northern Greece, presenting many risks for the construction of pavements. In a new classification system, it is possible for soils with homogeneous technical attributes to be distinguished in categories, which they will be based on the activity of the clayey phase -more specifically on the total specific area, that is, on the methylene blue value- in combination with their grain-size gradation.

The methylene blue test has been found to be an easy and very repeatable test method. Because of their simplicity, the suitability controls which are supported by the laboratorial tests, can be also conveyed in field testing.

The correlation of values of attributes obtained from the tests in methylene blue with the respective values of the plasticity index, the soil gradation (clay fraction, percent of material passing the No. 200 sieve) and with the sand equivalent showed that linearity exists in these relations and that the correlation coefficients were particularly high. For the relations to merit general application, they should be confirmed with the repetition of experimental processes in many more samples from the region of Thrace.

\section{References}

[1] Kollaros, G. \& Athanasopoulou, A., The character and identification of swelling soils in road construction projects, Proc. International Symposium on Engineering Geology and the Environment IAEG, eds. Marinos, Koukis, Tsiambaos \& Stournaras Balkema: Athens, pp. 187-192, 1997.

[2] Tran, N.L. \& Millon-Devigne, P., L'essai au bleu de méthylène en turbidimétrique, Bulletin de l'Association Internationale de Géologie de l'Ingénieur, 29(1), pp. 453-456, 1984.

[3] Taylor, R.K., Cation exchange in clays and mudrocks by methylene blue. $J$. Chem. Tech. Viotechnol., 35A, pp. 195-207, 1985.

[4] Sweere, G.T.H. \& Galjaard, P.J., The methylene blue test as a rapid means of estimating the cation exchange capacity of soils. International Symposium of Geotechnical Aspects of Mass and Material Transportation, Bangkok, pp. 47-52, 1984.

[5] Odell, R.T., Thornburn, T.H. \& McKenzy, L., Relationships of Atterberg limits to some other properties of Illinois Soils. Proceedings of the Soil Science Society of America, 24(5), pp. 297-300, 1960.

[6] Xeidakis, G.S., Assessment of the engineering and other properties of expansive soils by various methods. Ph.D. Thesis, Dept. of Civil Engineering, University of Leeds, England, 407 p., 1979.

[7] Farrar, D.M. \& Coleman, J.D., The correlation of surface area with other properties of 19 British clay soils. Journal of Soil Science, 18(1), pp. 118$124,1967$. 
[8] Nikolaides, A., Manthos, E. \& Sarafidou, M., Sand equivalent and methylene blue value of aggregates for highway engineering. Foundations of civil and environmental engineering 10, Publishing House of Poznan University of Technology, Poznan, pp 111-121, 2007.

[9] Petkovšek, A., Maček, M., Pavšič, P. \& Bohar, F., Fines characterization through the methylene blue and sand equivalent test: comparison with other experimental techniques and application of criteria to the aggregate quality assessment, Bull Eng Geol Environ 69, pp. 561-574, 2010. 